\title{
Numerical Simulation of Reoxidation Processes
}

\author{
Marek Bruna ${ }^{l, *}$, and Marek Galčík ${ }^{l}$ \\ ${ }^{1}$ University of Žilina, Faculty of Mechanical Engineering, Department of Technological Engineering, \\ Žilina, Slovakia
}

\begin{abstract}
Reoxidation is one of the main problem accompanying the aluminium alloy casting process. The oxide layer created on the melt surface during reoxidation is entrained into the bulk of liquid metal and "bifilms" are created. Bifilms have negative impact on cast quality and internal homogenity of final casting. Paper aim is to clarify the reoxidation phenomenon by visualization with the aid of ProCAST numerical simulation software. Experiemtnal work deals with the design of several types of gating systems (non pressurized and naturally pressurized) with vortex elements in order to determine how these elements affect the reoxidation processes. Achieved results clearly confirmed the positive effect of the naturally pressurized gating system with vortex elements. The evaluation focuses mainly on melt velocity and amount of oxides created in gating system and in mold cavity.
\end{abstract}

\section{Introduction}

The increasing demands on the aluminum alloys quality are related to the effort to eliminate as many casting defects as possible. One of the biggest problems in the production of castings from aluminum alloys is the reoxidation, which significantly affects the quality of liquid metal $[1,2]$.

Liquid metal reacts with the surrounding atmosphere and oxide layer of $\mathrm{Al}_{2} \mathrm{O}_{3}$ is formed on its surface. Reoxidation is considered to be the secondary oxidation that occurs from the beginning of the casting process until the moment when the mold cavity is filled, as well as the tertiary oxidation that occurs during solidification $[2,3]$.

The result of reoxidation processes are „bifilms“, which are formed and entrainment into the bulk of liquid metal by turbulence. Due to their small and compact dimensions, bifilms can pass through the entire gating system, they often cannot be removed even with the use of the filtration media. After the filling of mold cavity, when the melt is relatively calm, bifilms can grow and thus regain their original dimensions and act as nucleation sites for various casting defects. The quality of castings is also affected by the quality of the input material used. However, if turbulence occurs during the filling of the mold, even a good quality batch material will not prevent the formation of ,bifilms“ [4].

\footnotetext{
* Corresponding author: marek.bruna@fstroj.uniza.sk
} 
The amount of oxides and the melt flow velocity are among the most important parameters during the filling of the gating system, which affect the final quality of the casting. According to prof. Campbell [1], the critical melt velocity in the gates $0.5 \mathrm{~m} . \mathrm{s}^{-1}$. Higher velocity causes turbulence to occur, which consequently increases the amount of entrained bifilms. The formation of bifilms is also influenced by a poorly designed geometry of the gating system $[5,6]$.

By using a properly designed vortex elements as a part of gating system, flow velocity can be decreased to acceptable values [7].

Nowadays, there are very few studies on vortex elements and their effectiveness. Experimental work deals with the design of several types of gating systems (non pressurized and naturally pressurized) with using vortex elements in order to determine how these elements affect the melt velocity and the amount of oxides in the casting by using numerical simulation.

\section{Experimental procedures}

Construction of the gating system is commonly divided into pressurized and non-pressurized gating systems, based on the so-called 'choke area'. These constructions designs are not sufficient in terms of suppressing the reoxidation. Therefore, it was necessary to design a new type of gating system - naturally pressurized. The concept of a naturally pressurized gating system minimizes (or eliminates) the presence of turbulence. The system is designed without the 'choke area' as close as possible to 1:1:1 ratio, so that the melt is in all places in direct contact with the mold walls (except for the head area of flow) by natural backpressure in gating system due to friction resistance. Such a design is preferable because of the area exposed to further reoxidation is significantly smaller and it promotes the natural melt flow. Although this concept has been known for a long time, its use in practice is rare. The critical velocity of the melt flow in which turbulences will occurs $\left(0.5 \mathrm{~m} \cdot \mathrm{s}^{-1}-\right.$ the value specified by the researcher community for many alloys), especially when the melt enters through the gates is exceeded and splashes causes extensive reoxidation of molten metal. To reduce the melt velocity in naturally pressurized systems, the filters, various extensions of the runner or socalled vortex elements were used in previous research works. Based on the literary analysis it appears that the satisfactory results will be achieved by ensuring the combination of all these elements. The experimental work is focused on the numerical simulation by ProCAST software. Four types of gating systems were designed (Figure 1).

a)

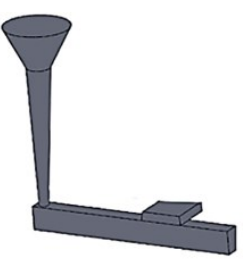

b)

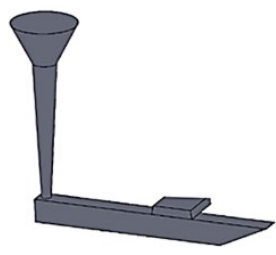

c)

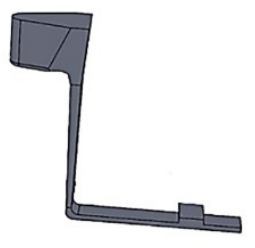

d)

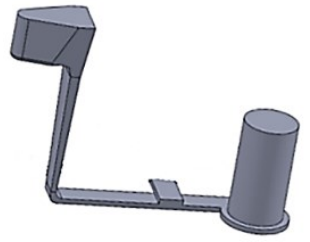

Fig. 1. Gating system design for experimental work a) non-pressurized gating system, b) nonpressurized gating system with modification of the runner, c) naturally pressurized gating system, d) naturally pressurized gating system with vortex extension.

Two systems are common concepts of the non-pressurized gating system (typically used for aluminium alloys), where one have modification of the runner which should lower the reoxidation (Figure 3b) and one is without modification (Figure 3a). Following two systems are based on the concept of the naturally pressurized gating system (Figure 3c), where one includes the modification of the runner as shown at Figure 3d. 
For the purposes of experiments, two types of castings were used. First casting design was intended for mechanical properties evaluation (tensile strength and elongation values in further experiments will be validated), exact dimensions are shown at Figure 2 (left). Second casting shape is related to further hot tear index evaluation, each bar have a different length which affects susceptibility to hot tearing Figure 2 (right).
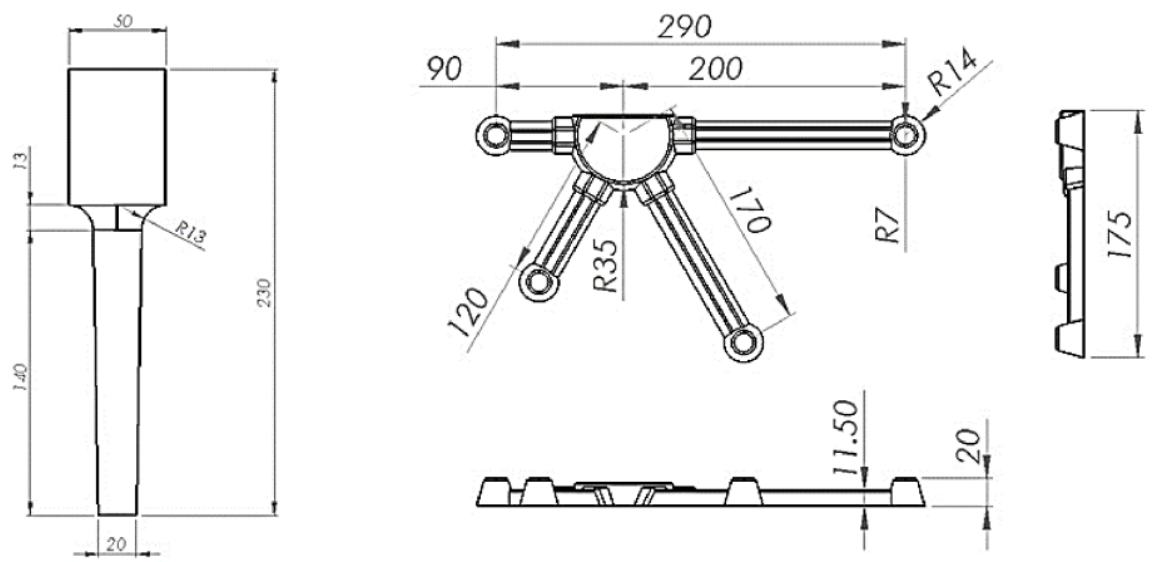

Fig. 2. Design of castings a) Mechanical properties b) Hot tear index.

\section{Simulations, results and discussion}

The numerical simulations, analysis of oxides and melt velocity during molds filling were evaluated. Casts were poured by gravity sand casting method (green sand). The melt temperature was $720 \pm 5{ }^{\circ} \mathrm{C}$ poured to a mold with temperature $20{ }^{\circ} \mathrm{C}$. The primary AlSi7Mg0.3 alloy was used from database without any modification.

\subsection{Analysis of turbulence energy}

The tracing indicator for turbulence energy was set to $\left[100 \mathrm{~cm}^{2} \cdot \mathrm{s}^{-2}\right]$. Based on the obtained results of the numerical simulation (Figure 3), the hypothesis that a non-pressurized gating system is insufficient in terms of reoxidation processes can be confirmed.
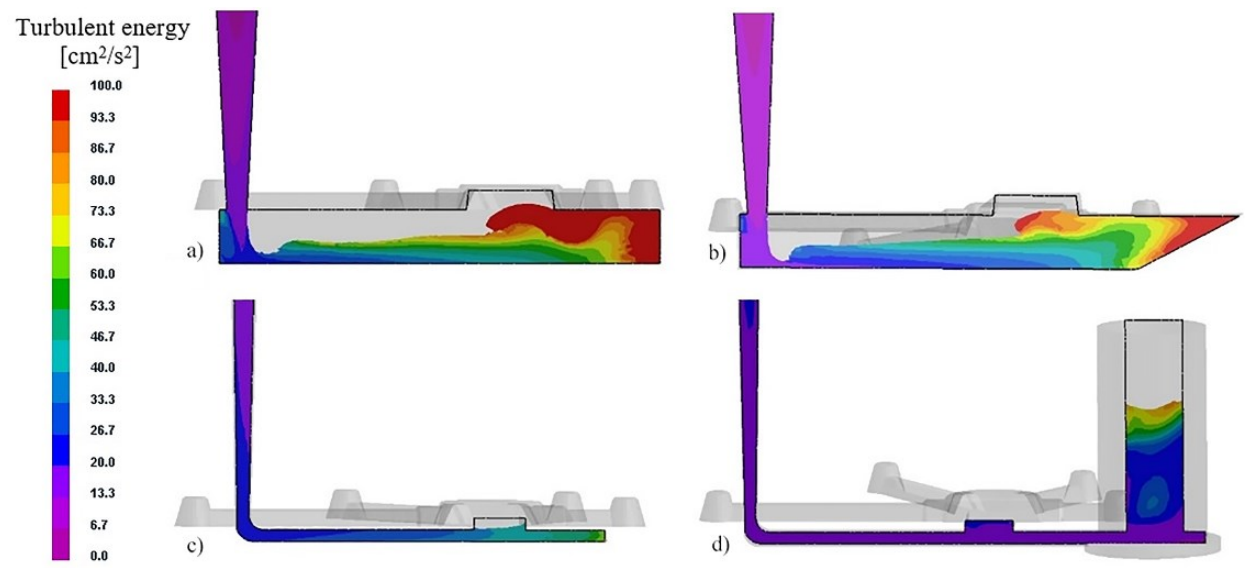

Fig. 3. Result of the turbulence energy analysis. 
As a consequence of imperfectly filled runner, a large space for surface layer entraining is available, also bounce wave can occur at the end of the runner. The result of the first variant (Figure 3a) shown that the melt suddenly bounce back, and the two surfaces are subsequently folded and joined, undeniably creating a large amount of bifilms. In the variant with modification in form of the gradual narrowing of the runner (Figure $3 b$ ), there was a slight suppression of bouncing wave, but the area exposed to further oxidation remains extensive. In the case of a naturally pressurized gating system (Figure $3 \mathrm{c}$ ), the place for turbulence is eliminated by a perfectly filled space in every point of the system. The critical place of this concept is the gate area because of the melt high velocity without any slowing mechanism, which will be analysed further. The best result was achieved in the naturally pressurized gating system with vortex extension of the runner (Figure 3d). The melt flow was directed to a vortex element, which ensures calm continuity of flow in the runner. Turbulent energy is situated in the vortex element and other parts of the filling system are protected from the negative influence of reoxidation.

\subsection{Analysis of the melt velocity}

The velocity of the melt flow affects the character and scale of turbulence present during the gating system filling. The result of the melt flow rate analysis $\left[\mathrm{m} . \mathrm{s}^{-1}\right]$ for the non-pressurized gating system, showed a reduction of the melt velocity in the choke area (Figure 4a, b), which ensures calm filling in the gate area. However, the critical area of further reoxidation and the presence of the bouncing wave in the runner remains problematic.

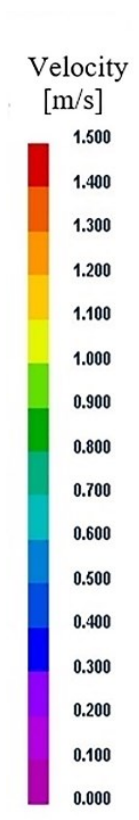

a)
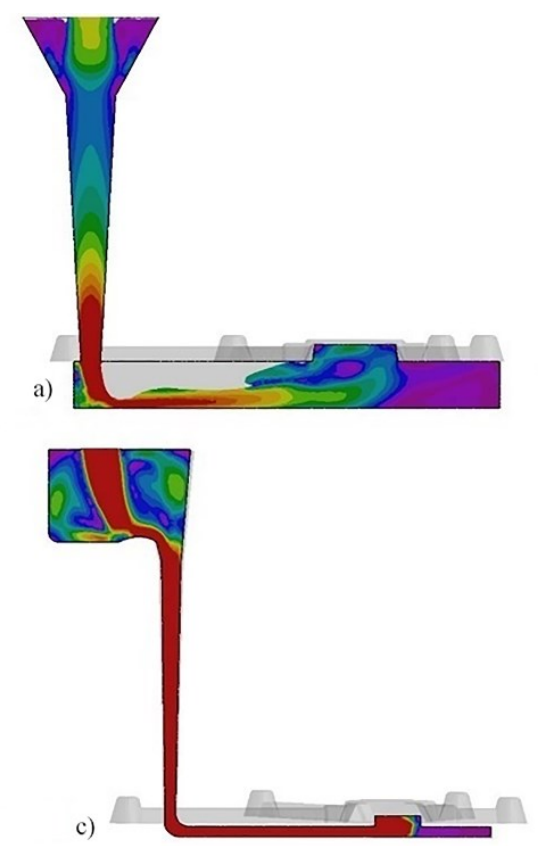
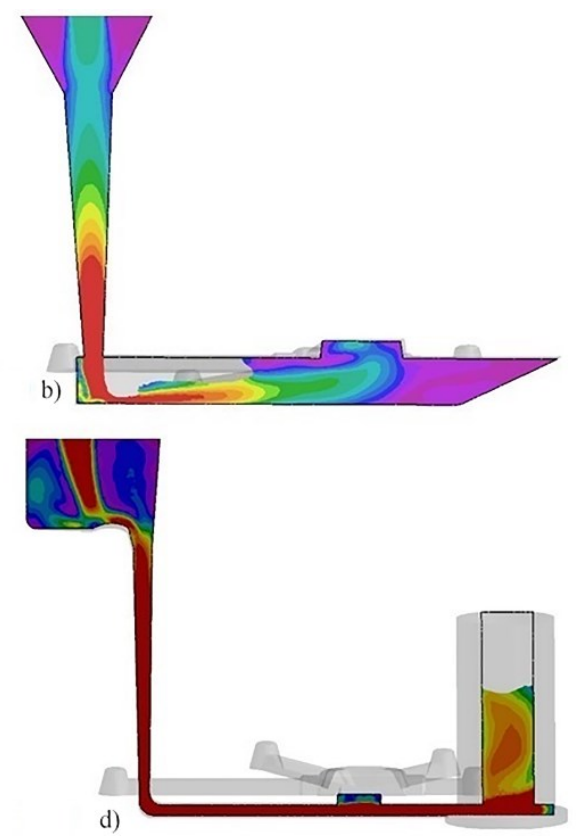

Fig. 4. Analysis of the melt flow velocity.

As mentioned before, in the naturally pressurized gating system is the critical velocity of the melt exceeded due to the absence of a mechanism to reduce it. Velocity energy at the end of the runner is transferred to the gate area (Figure 4c), where the splashes occurs, resulting in the extensive reoxidation. At this point, all benefits of a perfectly filled gating system during the filling are lost. Numerical simulations have shown the best results as the concept 
of naturally pressurized gating system with runner modification (Figure 4d). The energy of the melt flow is situated in the vortex element, which ensures a significant reduction of the melt velocity in the runner and also avoiding the splashes formation at the point of entry to the cast part of mold cavity. To better illustrate the described phenomenon occurring in the naturally pressurized gating system, melt spatter is shown on the casting design for the mechanical properties evaluation (Figure 5).

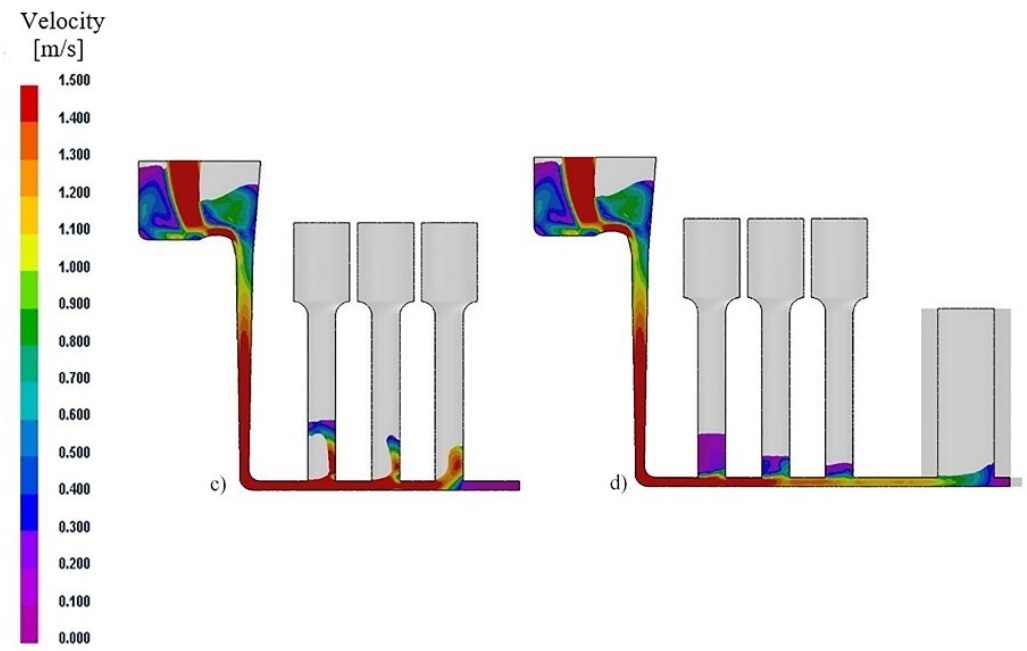

Fig. 5. Ingate velocity analysis.

\section{Further research}

Further research will be focused on combination of vortex elements and foam filters in mutual interaction. First step of next set of experimental works was already carried out and includes a proposal for optimal gating system design based on simulations. Several publications [8] suggest to use foam filters as a mean to reduce the melt velocity, first experiments supports this claims. Figure 6 a) shows velocity evaluation by vectors, where filter $(50 \times 50 \times 20 \mathrm{~mm}$, $30 \mathrm{ppi}$ ) is placed as a gate above the runner in horizontal position. The result of such a design is calm filling with velocity significantly below the critical value (no splashing, breaking waves and entrainemnt of the surface oxides). Figure $6 \mathrm{~b}$ ) represent the vortex gate design with foam filter (diameter $80 \mathrm{~mm}$, thickness $15 \mathrm{~mm}, 30 \mathrm{ppi}$ ) placed inside the gate. Results from the velocity point of view are promising, further evaluation is necessary, mainly by real casts production.
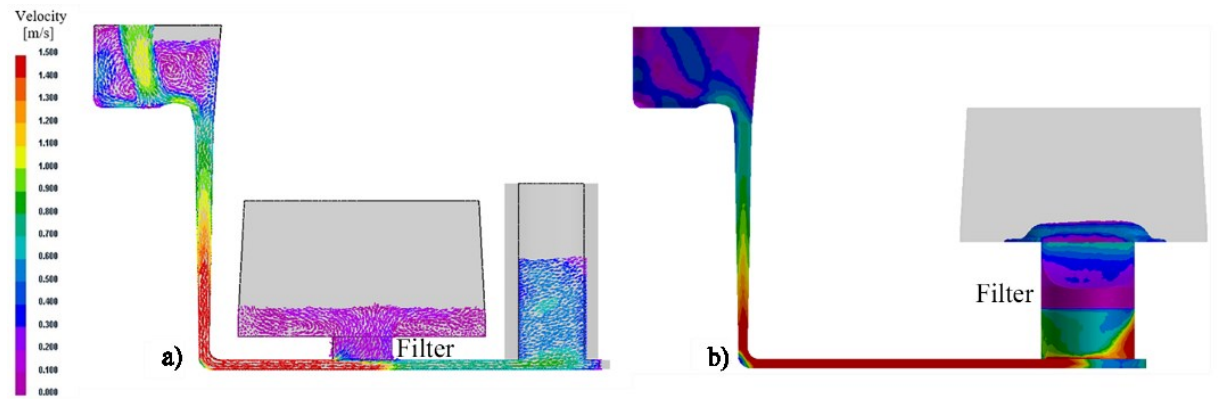

Fig. 6. Vortex elements in combination with foam filters. 


\section{Conclusion}

- Main reasons of bifilm occurrence are melt high velocity, imperfectly filled gating system and associated turbulence. As a result of these phenomena, the surface oxide layer is folded and entrained into the bulk of molten metal.

- Numerical simulation proven that filling systems molded to follow the shape of the falling stream (naturally pressurized system) are successful to reduce the conditions for forming entrainment defects.

- Using of vortex element reduces the critical velocity of flow in which the melt enters the mold cavity through the gate, and also minimizes the oxide occurrence in the castings. The front head of molten metal enters the cylindrical end, where is the flow directed to a tangential gradient eddying motion.

Acknowledgment: This article was made under support of project V-1/0706/18 Concept and realization of modern centre of diagnostics and quality control for castings and weldments.

\section{References}

1. J. Campbell. Complete casting handbook $2^{\text {nd }}$ edition. Oxford, Elsevier Ltd. (2015)

2. E. Erzi, Ö. Gürsoy, C. Yüksel, M. Colak, D. Dispinar, Metals. 9, 957, (2019)

3. D. Bolibruchova, R. Podprocka, R. Pastircak, et al. The role of Mn in aluminium alloys with a higher iron content. Archives of metallurgy and materials. Vol. 63, Issue: 4, Pp. 1883-1888, (2018)

4. A. Sladek, M. Patek, M. Mician, Archives of Metallurgy and Materials, 62 (3), pp. 1597 1601 (2017)

5. Major-Gabrys, K. 2015. Biodegradable materials as foundry moulding sands binders. METALURGIJA. Vol. 54, Issue 3 , Pp. 591-593, (2015)

6. R. Lenhard, K. Kaduchová, Š. Papučík, J. Jandačka. Utilization of heat pipes for transfer heat from the flue gas into the heat transfer medium. EPJ web of conferences [online]., 67, 02067 [cit. 2020-09-07]. ISSN 2101-6275, (2014)

7. Š. Papučík, R. Lenhard, K. Kaduchová, J. Jandačka, J. Koloničný, J. Horák. Dependence the amount of combustion air and its redistribution to primary and secondary combustion air and his depending on the boiler [online]. 1608 (1), 98-102. ISSN 0094243X, (2014)

8. R. Dojka, J. Jezierski, J. Campbell. Optimized Gating System for Steel Castings. Journal of Materials Engineering and Performance. 10, (2018) 